University of Nebraska - Lincoln

DigitalCommons@University of Nebraska - Lincoln

2013

\title{
Micromechanical analysis of nanoparticle-reinforced dental composites
}

\author{
Yi Hua \\ University of Nebraska-Lincoln, yhua3@unl.edu \\ Linxia Gu \\ University of Nebraska-Lincoln, gul@fit.edu \\ Hidehiko Watanabe \\ University of Nebraska Medical Center, hwatanabe@unmc.edu
}

Follow this and additional works at: https://digitalcommons.unl.edu/mechengfacpub

Part of the Biology and Biomimetic Materials Commons, Biomedical Engineering and Bioengineering Commons, Dental Materials Commons, and the Equipment and Supplies Commons

Hua, Yi; Gu, Linxia; and Watanabe, Hidehiko, "Micromechanical analysis of nanoparticle-reinforced dental composites" (2013). Mechanical \& Materials Engineering Faculty Publications. 94.

https://digitalcommons.unl.edu/mechengfacpub/94

This Article is brought to you for free and open access by the Mechanical \& Materials Engineering, Department of at DigitalCommons@University of Nebraska - Lincoln. It has been accepted for inclusion in Mechanical \& Materials Engineering Faculty Publications by an authorized administrator of DigitalCommons@University of Nebraska Lincoln. 


\title{
Micromechanical analysis of nanoparticle-reinforced dental composites
}

\author{
Yi Hua, ${ }^{1}$ Linxia Gu, ${ }^{1,2}$ and Hidehiko Watanabe ${ }^{3}$ \\ 1. Department of Mechanical and Materials Engineering, \\ University of Nebraska-Lincoln, Lincoln, NE 68588-0656 \\ 2. Nebraska Center for Materials and Nanoscience, Lincoln, NE 68588-0656 \\ 3. Department of Adult Restorative Dentistry, University of Nebraska Medical Center, \\ College of Dentistry, Lincoln, NE 68583-0750 \\ Corresponding author - L. Gu, Department of Mechanical and Materials Engineering, \\ University of Nebraska-Lincoln, Lincoln, NE 68588-0656, USA; tel 402 472-7680, fax 402 472-1465, email lgu@unl.edu
}

\begin{abstract}
The mechanical behavior of $\mathrm{TiO}_{2}$ nanoparticle-reinforced resin-based dental composites was characterized in this work using a three-dimensional nanoscale representative volume element. The impacts of nanoparticle volume fraction, aspect ratio, stiffness, and interphase zone between the resin matrix and nanoparticle on the bulk properties of the composite were characterized. Results clearly demonstrated the mechanical advantage of nanocomposites in comparison to microfiber-reinforced composites. The bulk response of the nanocomposite could be further enhanced with the increased nanoparticle volume fraction, or aspect ratio, while the influence of nanoparticle stiffness was minimal. The effective Young's modulus and yield strength of the composite was also significantly affected by the interphase stiffness. Results obtained in this work could provide insights for the optimization of nanoparticle-reinforced dental composites.
\end{abstract}

Keywords: dental nanocomposites, representative volume element, sensitivity, interphase

\section{Introduction}

Resin-based composites (RBCs) are currently among the most popular dental restorative materials due to their good aesthetic properties (Xia, Zhang, Xie, \& Gu, 2008). Compared to dental alloys and ceramics, the application of RBCs to posterior teeth is still restricted to some extent due to their inferiority in wear resistance, fracture toughness and shrinkage behavior as well as bond longevity of dentin bonding agents. Recently various nanoparticles are used to improve the performance of RBCs and the results show that desired property enhancements can be achieved in these composites with small amounts of nanoparticles (Turssi, Ferracane, \& Ferracane, 2006; Wetzel, Rosso, Haupert, \& Friedrich, 2006; Yu, Ahn, Lim, \& Lee, 2009). The mechanical behavior of nanocomposite is regulated by its microstructures such as nanoparticle volume fraction, aspect ratio and stiffness. In addition, the interphase zone at the nanoparticle/matrix interface raised increased attention. The interphase is the region formed between nanoparticle and matrix, due to altered molecular structure of the resin matrix at the interface with nanoparticles (Eitan, Fisher, Andrews, Brinson, \& Schadler, 2006; Zhang, Zhang, Friedrich, \& Eger, 2006). The role of interphase is not fully understood due to controversial results in the literature. $\mathrm{Yu}$ et al. reported that an interphase zone within a $\mathrm{Al}_{2} \mathrm{O}_{3}$ nanoparticle-reinforced epoxy was stiffer than the matrix (Yu, Yang, \& Cho, 2009). On the contrast, Odegard et al. stated that the interphase within a silica nanoparticle/polyimide composite was softer than the matrix (Odegard, Clancy, \& Gates, 2005). Another challenge is to quantify the interfacial bonding strength between resin matrix and nanoparticles. Due to complicated properties of the interphase zone, weak bonding areas are quite likely to exist and composite damage may occur at a lower loading. A systematic study on the role of interphase including its damage behavior 
on the bulk behavior of the composite is needed, especially for nanoparticle-reinforced dental composites (Barbero, Abdelal, \& Caceres, 2005; Lee, Chiang, Lin, Huang, \& Dong, 2000).

In this study, a nanoscale representative volume element (RVE) was used to predict the mechanical behavior of $\mathrm{TiO}_{2}$ nanoparticle-reinforced resin-based dental composites and the results were validated against that obtained by the non-interaction approximation (NIA). The mechanical advantage of this nanoparticle-reinforced composites compared with that reinforced by glass fibers was illustrated. The influences of nanoparticle volume fraction, aspect ratio and stiffness were examined in terms of effective Young's modulus and yield strength of the composite. In addition, the interphase zone created by alternated dynamics of resin matrix molecules in the vicinity of the nanoparticles was considered. The effect of interphase stiffness on the bulk properties of the composite was evaluated. Cohesive material was used to the interphase and the damage at interphase was investigated. The results of the investigation are expected to provide some design parameters for the microstructural optimization of nanoparticle-reinforced dental composites.

\section{Finite element modeling}

The microstructure of the $\mathrm{TiO}_{2}$ nanoparticle-reinforced resin-based dental composites is represented by a three-dimensional RVE (100 nm each side), as shown in Figure 1(a). Total of 30 identical $\mathrm{TiO}_{2}$ nanoparticles are assumed as spheres and randomly dispersed. The nanoparticle centers are generated using the random sequential adsorption algorithm (Widom, 1966), in which the probability of finding a nanoparticle at a given position is the same in all directions. The nanoparticle diameter depends on the nanoparticle volume fraction and aspect ratio. In the baseline model, the nanoparticle volume fraction $\left(V_{p}\right)$ is set as $5 \%$, and aspect ratio $\left(A_{r}\right)$ is chosen as 1 . Then the nanoparticle diameter is calculated as $14.7 \mathrm{~nm}$. Lower volume fraction and larger aspect ratio result in a smaller nanoparticle diameter. The material properties of $\mathrm{TiO}_{2}$ nanoparticles are adopted with Young's modulus $E_{p}=282.76$ GPa and Poisson's ratio $v_{p}=0.3$ (Sivasankaran, Sivaprasad, Narayanasamy, \& Iyer, 2010). The resin matrix of urethane dimethacrylate (UDMA) monomer is taken from the published experimental test as $E_{m}=3.9 \mathrm{GPa}, v_{m}=0.3$ and yield strength, defined as the stress at which material would experience plastic flow, $\sigma_{y}=60 \mathrm{MPa}$ (Lassila, Nohrstrom, \& Vallittu, 2002). The two phases are meshed with 4-node tetrahedral linear element (C3D4), as shown in Figure 1(b). A mesh convergence study is conducted and the minimum mesh size of $0.5 \mathrm{~nm}$ is chosen. Periodic boundary conditions are imposed to the RVE faces. A uniform $3 \%$ strain is applied to the model along the $x$-direction.

\section{Cohesive zone material model}

A cohesive zone material model (ABAQUS) is adopted to simulate the damage behavior in the interphase region. This model is implemented via cohesive elements, including a linear traction-separation law, which relates the stress vector to the strain vector across the cohesive zone. Both stress and strain vectors have three components in three directions, one component normal to the surface and two shear components. Before damage initiation, the traction-separation relationship is given by

$$
t=\left\{\begin{array}{c}
t_{n} \\
t_{s} \\
t_{t}
\end{array}\right\}=\left[\begin{array}{ccc}
K_{n n} & K_{n s} & K_{n t} \\
K_{n s} & K_{s s} & K_{s t} \\
K_{n t} & K_{s t} & K_{t t}
\end{array}\right]\left\{\begin{array}{c}
\varepsilon_{n} \\
\varepsilon_{s} \\
\varepsilon_{t}
\end{array}\right\}=\mathrm{K} \varepsilon
$$

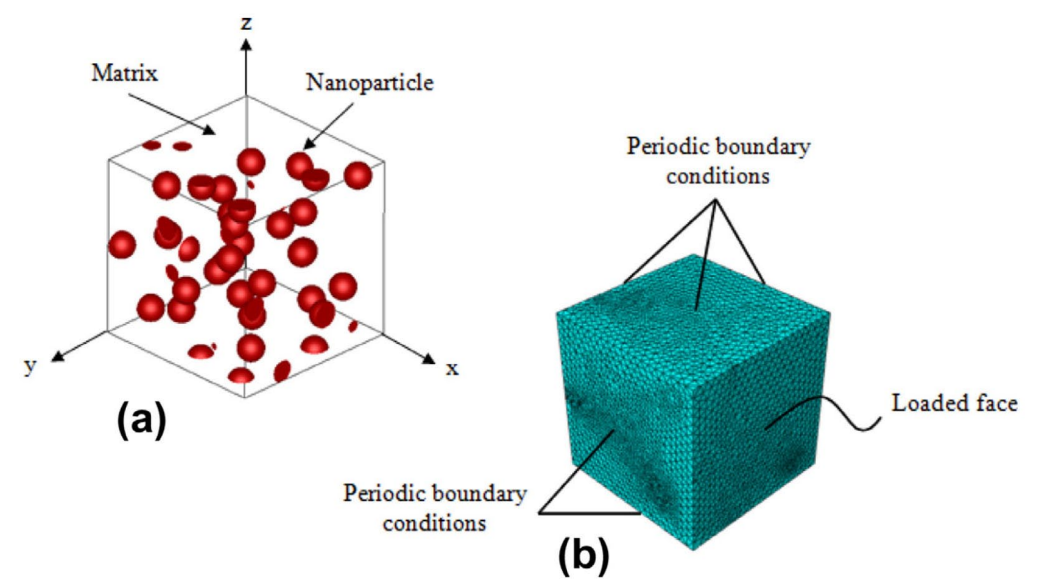

Figure 1. Typical (a) geometry and (b) mesh of a three-dimensional RVE with random distributed sphere-shaped nanoparticles. 
where $\mathrm{K}$ is the interphase stiffness matrix. The coupled behavior between the normal and shear components is not considered in this work, thus the off-diagonal terms in the interphase stiffness matrix in the above expression will always be 0 . The strain vector $\varepsilon$ is defined as

$$
\varepsilon_{n}=\frac{\delta_{n}}{T_{0}}, \quad \varepsilon_{s}=\frac{\delta_{s}}{T_{0}}, \quad \varepsilon_{t}=\frac{\delta_{t}}{T_{0}}
$$

with $\delta_{n}, \delta_{s}$, and $\delta_{t}$ being the separations and $T_{0}$ the initial interphase thickness.

Damage initiation is predicted using a maximum nominal stress criterion

$$
\max \left\{\frac{\left\langle t_{n}\right\rangle}{t_{n}^{0}}, \frac{t_{s}}{t_{s}^{0}}, \frac{t_{t}}{t_{t}^{0}}\right\}=1
$$

where the symbol \langle\rangle signifies that only positive values are taken into account and $t^{0}{ }_{i}$ is the maximum traction in each separation mode.

Damage evolution is represented by a single scalar parameter $D$, which is calculated based on displacement with linear softening

$$
D=\frac{\delta_{m}^{f}\left(\delta_{m}^{\max }-\delta_{m}^{0}\right)}{\delta_{m}^{\max }\left(\delta_{m}^{f}-\delta_{m}^{0}\right)}
$$

where $\delta_{m}{ }^{f}$ is the effective separation at failure, $\delta_{m}{ }^{0}$ is the separation at initiation of damage and $\delta_{m}$ max is the maximum separation attained in the complete loading history. The damage variable $D$ varies in the range of $[0,1]$. Its value is 0 for undamaged material and 1 for failed material. The stress components of the traction-separation relationship are affected by this parameter in the following way

$$
\begin{aligned}
& t_{n}= \begin{cases}(1-D) \bar{t}_{n}, & \bar{t}_{n} \geq 0 \\
\bar{t}_{n}, & \text { otherwise }\end{cases} \\
& t_{s}=(1-D) \bar{t}_{s} \\
& t_{t}=(1-D) \bar{t}_{t}
\end{aligned}
$$

\section{Results and discussion}

\subsection{Validation}

The effective Young's modulus of the composite predicted by the finite element (FE) simulation of a RVE of the microstructure has been validated against that obtained by the NIA, in which the interactions between inclusions are neglected. The principle of this scheme is that the average strain in the inclusions of a two-phase material could be estimated by the solution for a single inclusion embedded in an infinite medium subjected to a remote uniform stain within the matrix. Specifically, for a RVE domain $\omega$ contains a matrix phase (denoted by the subscript 0 ) and an inclusion phase (denoted by the subscript 1), the mean strain over all inclusions is related by

$$
\langle\varepsilon\rangle_{\omega_{1}}=B^{\varepsilon}:\langle\varepsilon\rangle_{\omega_{0}}
$$

where a colon designates a tensor product contracted over two indices and the brackets \langle\rangle represent a volume average. The strain concentration tensor $B^{\varepsilon}$ is given by Pierard, Friebel, \& Doghri, (2004)

$$
\left.B^{\varepsilon}=H^{\varepsilon}\left(I, C_{0}, C_{1}\right)=\left\{I+\xi_{\left(I, c_{0}\right)}:\left(C_{0}\right)^{-1}: C_{1}-I\right]\right\}^{-1}
$$

where $I$ is the fourth-order symmetric identity tensor, $C_{i}$ is the stiffness of each phase, $\xi_{\left(I_{1}, c_{0}\right)}$ is Eshelby's tensor and depends on the geometry of inclusion and matrix stiffness.

The effective bulk stiffness $\bar{C}$ can then be expressed as

$$
\bar{C}=\left[V_{1} C_{1}: B^{\varepsilon}+\left(1-V_{1}\right) C_{0}\right]:\left[V_{1} B^{\varepsilon}+\left(1-V_{1}\right) I\right]^{-1}
$$

where $V_{i}$ is the volume fraction of each phase and $V_{0}+V_{1}=1$.

A comparison of the results from the NIA and FE model is shown in Figure 2. It is observed that the effective Young's modulus predicted by the FE model agreed very well with that calculated by the NIA, with a maximum deviation of only $1.6 \%$. A similar comparison has also been observed for the case of randomly oriented nanoclay-reinforced polymer matrix subjected to tensile loading (Hbaieb, Wang, Chia, \& Cotterell, 2007). 
Figure 2. Comparison between NIA and FE models.

Figure 3. Comparison of the reinforcement of resin matrix by nanoparticles and glass fibers.
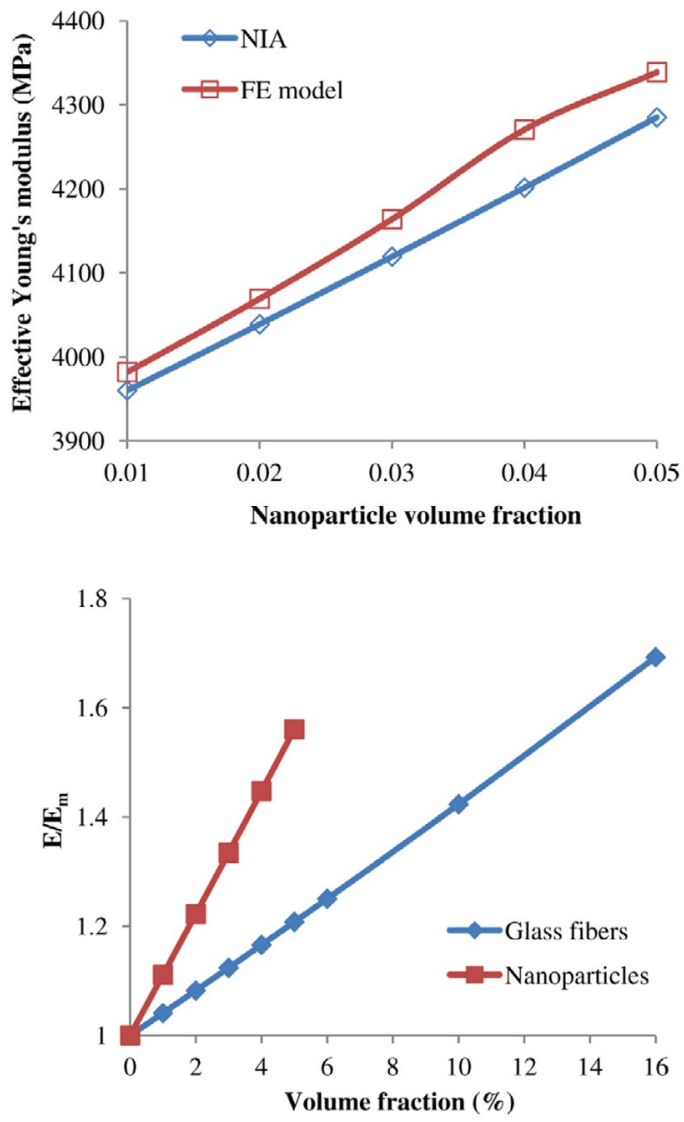

\subsection{Mechanical advantage of nanocomposites}

Nanocomposites are of great interest because they offer exceptional reinforcement at very low nanoparticle volume fraction (Ferracane, Jan 2011). In this section, the effective Young's modulus of dental composites reinforced by $\mathrm{TiO}_{2}$ nanoparticles has been compared with that reinforced by glass fibers, as demonstrated in Figure 3. The material properties of glass fibers are adopted with Young's modulus $E=72.4$ GPa and Poisson's ratio $v$ $=0.2$ (Tucker \& Liang, 1999). For consistence, the aspect ratio of both $\mathrm{TiO}_{2}$ nanoparticles and glass fibers was set as 50. It was observed that the effective Young's modulus of dental composites increased much more rapidly by addition of nanoparticles than by glass fibers. Specifically, increasing 30\% composite modulus required approximately 3\% volume fraction of nanoparticles; whereas, twice this amount of glass fibers was needed to achieve the same stiffness enhancement. This mechanical advantage could be attributed to the large specific surface area of nanoparticles, which could greatly facilitate the transfer of load from resin matrix to nanoparticles, leading to higher stiffness and better wear resistance than microcomposites.

\subsection{Characterization of dental composites}

In this section we consider predictions for dental composites containing nanoparticles in the arrangement shown in Figure 1 with two phases only: the nanoparticles and the matrix. Table 1 lists the effective Young's modulus and yield strength of the composite with various nanoparticle volume fractions under the condition of having fixed nanoparticle stiffness of $282.76 \mathrm{GPa}$ and aspect ratio of 1 . It was observed that the effective Young's modulus and yield strength of the composite increased almost linearly with the increase of nanoparticle volume fraction from $1 \%$ to $5 \%$. By adding $5 \%$ volume fraction of nanoparticles to the matrix, the effective Young's modulus and yield strength of the composite could increase $9.9 \%$ and 5.0\%, respectively. However, the sensitivity of composite to the particle volume fraction depended on the dispersion of particles, especially at high volume fraction, which also positively correlated with the mass fraction of nanoparticles. Tian et al. (2008) found that 1-3 wt.\% of nanoparticles was the best loading ratio for enhanced mechanical strengths of dental composites due to the optimal dispersion. Larger mass fraction of nanoparticles may even have adverse effects on the mechanical properties. 
To investigate the effect of nanoparticle aspect ratio on the bulk properties of the composite, five RVEs with various nanoparticle aspect ratios $\left(A_{r}=1,5,15,30\right.$, and 50) were considered here under the condition of having fixed nanoparticle stiffness of $282.76 \mathrm{GPa}$ and volume fraction of $5 \%$. The comparative results were shown in Table 2. Nanoparticles with larger aspect ratio proved to be more efficient in composite stiffness enhancement. There was a maximum $32.5 \%$ increase in the effective Young's modulus when nanoparticle aspect ratio increased from 1 to 30 . The overall increase rate of composite stiffness reduced though with the larger nanoparticle aspect ratio. The effective Young's modulus of the composite increased only $7.2 \%$ when nanoparticle aspect ratio increased from 30 to 50 . The influence of nanoparticle aspect ratio on the variation of the effective yield strength of the composite has a similar increasing trend. There was a maximum $35.2 \%$ increase in the effective yield strength when nanoparticle aspect ratio increased from 1 to 30 . The rate of increase was only $6.7 \%$ for nanoparticle aspect ratio increased from 30 to 50. This saturation of reinforcing effect for large nanoparticle aspect ratio was also known as the classic "shear-lag" behavior, which described the limiting effect of the small ratio of matrix modulus to particle modulus on the reduction of the load transfer efficiency between phases (Cox, 1952; Hua \& Gu, 2012).

For nanoparticles with a fixed aspect ratio of 1 and volume fraction of $5 \%$, the influence of nanoparticle stiffness (elastic modulus $E_{p}$ ranging from $3 E_{m}$ to $144 E_{m}$ ) on the mechanical behavior of the composite has been evaluated and the effective Young's modulus and yield strength are presented in Table 3. It was observed that the effective Young's modulus and yield strength of the composite were not sensitive to nanoparticle stiffness. There was only a marginal $5.1 \%$ and $2.0 \%$ increase in the effective Young's modulus and yield strength when nanoparticle modulus increased from $11.7 \mathrm{GPa}\left(3 E_{m}\right)$ to $561.6 \mathrm{GPa}\left(144 E_{m}\right)$. The growth rate in effective Young's modulus decreased with larger nanoparticle stiffness. For nanoparticle modulus larger than $35.1 \mathrm{GPa}$ $\left(9 E_{m}\right)$, the effective Young's modulus of the composite was almost unchanged. Fornes and Paul (2003) have demonstrated that increasing the particle stiffness could improve the reinforcement of composites, especially for particle aspect ratios greater than 20 30. Thus, the low particle aspect ratio $\left(A_{r}=1\right)$ used in this work was the main factor that influenced the reinforcement sensitivity of composites to nanoparticle stiffness.

\subsection{Effect of interphase}

The improved properties of dental composites are due not only to the loading amount, morphology and intrinsic properties of the nanoparticles alone, but also to the extensive interphase created from polymer mole-

Table 1. Comparison of effective Young's modulus and yield strength with different nanoparticle volume fractions $\left(E_{p}=282.76 \mathrm{GPa}, A_{r}=1\right)$.

\begin{tabular}{ccc}
\hline Volume fraction $(\%)$ & Young's modulus $(\mathrm{MPa})$ & Yield strength $(\mathrm{MPa})$ \\
\hline 1 & 3959.869 & 60.586 \\
2 & 4038.746 & 61.187 \\
3 & 4119.267 & 61.789 \\
4 & 4201.347 & 62.390 \\
5 & 4285.102 & 62.991 \\
\hline
\end{tabular}

Table 2. Comparison of effective Young's modulus and yield strength with different nanoparticle aspect ratios $\left(E_{p}=282.76 \mathrm{GPa}, V_{f}=5 \%\right)$.

\begin{tabular}{ccc}
\hline Aspect ratio (L/D) & Young's modulus $(\mathrm{MPa})$ & Yield strength $(\mathrm{MPa})$ \\
\hline 1 & 4285.102 & 62.991 \\
5 & 4446.259 & 65.360 \\
15 & 5055.152 & 75.069 \\
30 & 5678.667 & 85.180 \\
50 & 6061.867 & 90.928 \\
\hline
\end{tabular}

Table 3. Comparison of effective Young's modulus and yield strength with different nanoparticle elastic moduli $\left(A_{r}=1, V_{f}=5 \%\right)$.

\begin{tabular}{ccc}
\hline Particle modulus $\left(E_{p} / E_{m}\right)$ & Young's modulus $(\mathrm{MPa})$ & Yield strength $(\mathrm{MPa})$ \\
\hline 3 & 4082.970 & 61.857 \\
9 & 4209.562 & 62.512 \\
18 & 4250.842 & 63.125 \\
36 & 4273.197 & 62.816 \\
72 & 4285.102 & 62.991 \\
144 & 4291.088 & 63.079 \\
\hline
\end{tabular}


Figure 4. Schematic of nanoparticle and its surrounding interphase.
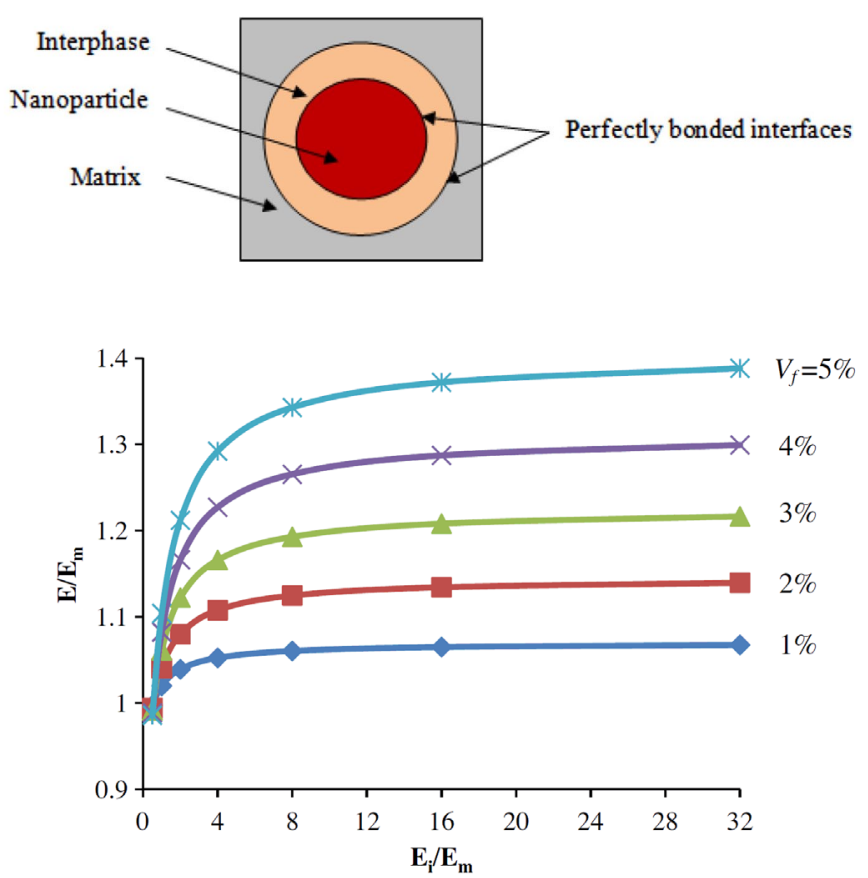

Figure 5. Effect of interphase stiffness on the effective Young's modulus of the composite with different nanoparticle volume fractions $\left(E_{p}=282.76 \mathrm{GPa}\right.$, $\left.A_{r}=1\right)$. cules of altered mobility in the vicinity of the nanoparticles (Fan, Tsui, Tang, \& Chow, 2004). Thus a second important consideration beyond pure reinforcement of the nanoparticle is the potential extent and influence of this interphase when characterizing the mechanical behavior of the nanocomposite. Figure 4 illustrates schematically the nanoparticle surrounded by an annular interphase of thickness $t_{i}$. In our baseline model with nanoparticle diameter of $14.7 \mathrm{~nm}$, the interphase thickness $t_{i}$ is set as $3.7 \mathrm{~nm}$. Then the ratio of interphase thickness to nanoparticle radius is about 0.5 , which is much larger than that of microparticle-filled composites, which is in the range of 0.02-0.07 (Iisaka \& Shibayama, 1978). Perfect bonding is assumed at the interfaces between nanoparticle and interphase and between interphase and matrix.

Figure 5 shows the influence of interphase stiffness $\left(E_{i}\right)$ on the effective Young's modulus of the composite with different nanoparticle volume fractions. The Poisson's ratio for the interphase is assumed to be equal to that of the matrix $\left(v_{i}=0.3\right)$. It is interesting to note that the effective Young's modulus of the composite was dependent on the interphase stiffness. For the composite with 1 vol\% nanoparticles, the effective Young's modulus of the composite increased $6.3 \%$ when interphase stiffness increased from $0.5 E_{m}$ to $8 E_{m}$. A significant increase was observed for the composite with higher nanoparticle volume fraction. There was a maximum $36.2 \%$ increase in the effective Young's modulus when interphase stiffness increased from $0.5 E_{m}$ to $8 E_{m}$ with a nanoparticle volume fraction of $5 \%$. This was due to an interphase of certain stiffness played a more prominent role in the composite with a higher nanoparticle volume fraction. For the interphase stiffness larger than 8 $E_{m^{\prime}}$ the effective Young's modulus of the composite was almost unchanged. The effective Young's modulus increased only $4.6 \%$ when interphase stiffness increased from $8 E_{m}$ to $32 E_{m}$ with the nanoparticle volume fraction of $5 \%$. It should be noted that stiffness variations across the interphase zone were reported (Lee, Wang, Pharr, \& Xu, 2007; Sevostianov \& Kachanov, 2006; Sevostianov \& Kachanov, 2007). This variation might change the effective Young's modulus of the nanocomposite, however, this alternation is minimal as illustrated by Sevostianov \& Kachanov (2006, 2007). Therefore the isotopic assumption of the interphase properties in this work is justified. Although only elastic stiffness is illustrated here, the interphase could have dramatically different thermal expansion (Lee et al., 2000; Wei, Srivastava, \& Cho, 2002) and viscoelastic properties (Fisher \& Brinson, 2001; Liu \& Brinson, Sep 2006) from the matrix, which would lead to markedly different global response of the nanocomposites.

As mentioned previously, damage may occur at very low level of loading in the interphase zone. In order to perform the damage analysis, three-dimensional 6-node linear triangular prism cohesive elements (COH3D6) were employed in this area. The maximum nominal stress criterion was used to control the initiation of the damage while the damage evolution was based on displacement with linear softening, as described in Section 3. Detailed parameters of the cohesive zone material model were listed in Table 4 . Figure 6 shows the contour plots of the scalar stiffness degradation (SDEG) in the RVE. It was seen that damage occurred at the outer edge of the interphase and progressively moved toward the inner edge. Note that the maximum principal strain field in the interphase was up to four times higher than that far away from the nanoparticle-matrix interface. This 


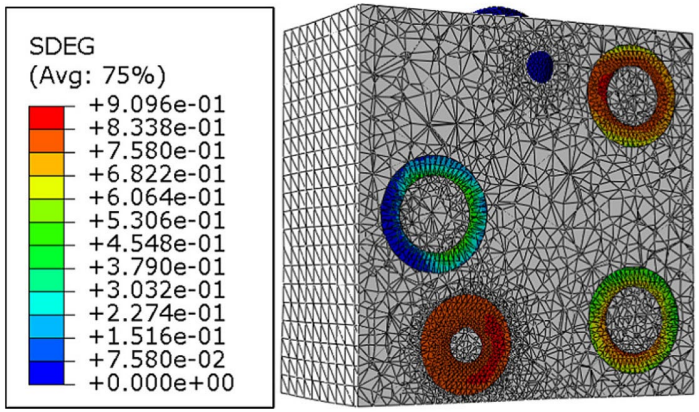

Figure 6. Contour plot of the damage level (SDEG, 0 indicated undamaged material, higher value indicated more damage) in the dental composites.

Table 4. Detailed parameters of the cohesive material properties.

Traction-separation behavior

Damage initiation: maximum nominal stress criterion

Damage evolution: based on displacement with linear softening
Normal stiffness: $K_{n n}=3900 \mathrm{MPa}$

Shear stiffness: $K_{t t}=K_{\mathrm{cs}}=1500 \mathrm{MPa}$

Normal-only mode: $t_{n}^{\delta_{S}^{S}}=60 \mathrm{MPa}$

Shear-only mode: $t_{s}^{0}=t_{t}^{0}=25 \mathrm{MPa}$

Displacement at failure (measured from damage initiation): $\delta_{m}{ }^{0}=5 \mathrm{~nm}$

implies a decreasing load carrying capacity in this area. Compared to the model without considering damage at interphase, the effective yield strength of the damageable model decreased $4.6 \%$ while the effective Young's modulus remained almost the same. The damageable model couldn't carry any more loads when the strain reached 0.026 .

\section{Conclusions}

The mechanical behavior of $\mathrm{TiO}_{2}$ nanoparticle-reinforced resin-based dental composites was investigated through the validated nanoscale RVE. The nanoparticle-reinforced composites demonstrated their exceptional reinforcement at very low nanoparticle volume fraction, compared with that reinforced by glass fibers. The influences of nanoparticle volume fraction, aspect ratio, and stiffness, as well as interphase stiffness, on the bulk properties of the composite were systematically evaluated. The damage behavior of the interphase zone was also investigated. The conclusions can be summarized as follows:

- The mechanical advantage of nanocomposites over microcomposites was demonstrated such that the reinforcement effect of the nanoparticle with $3 \%$ volume fraction on the stiffness is the same as the glass fiber with twice of the volume fraction.

- The effective Young's modulus and yield strength of the composite increased almost linearly with the increase of nanoparticle volume fraction from $1 \%$ to $5 \%$ considering perfect nanoparticle dispersion.

- The effective Young's modulus and yield strength of the composite was dependent on the interphase stiffness, while its sensitivity with respect to nanoparticle stiffness was minimal.

- Nanoparticles with larger aspect ratio could improve the bulk response of the composite. For nanoparticle aspect ratio larger than 30, the reinforcing effect reached saturation. These results could be used to enhance the fundamental understanding of mechanical behavior of nanoparticle-reinforced resin-based dental composites, and provide guidance for optimizing the compositions of dental composites.

\section{References}

ABAQUS v6.10 documentation (2011). Dassault Systèmes Simulia Corp; online at http://www.3ds.com/support/ documentation/v6-users-guide/

Barbero, E. J., Abdelal, G. F., \& Caceres, A. (2005). A micromechanics approach for damage modeling of polymer matrix composites. Composite Structures, 67, 427-436.

Cox, H. L. (1952). The elasticity and strength of paper and other fibrous materials. British Journal of Applied Physics, 3, 72-79.

Eitan, A., Fisher, F. T., Andrews, R., Brinson, L. C., \& Schadler, L. S. (2006). Reinforcement mechanisms in MWCNTfilled polycarbonate. Composites Science and Technology, 66, 1162-1173.

Fan, J. P., Tsui, C. P., Tang, C. Y., \& Chow, C. L. (2004). Influence of interphase layer on the overall elasto-plastic behaviors of HA/PEEK biocomposite. Biomaterials, 25, 5363-5373. 
Ferracane, J. L. (Jan 2011). Resin composite-state of the art. Dental Materials, 27, 29-38.

Fisher, F. T., \& Brinson, L. C. (2001). Viscoelastic interphases in polymer-matrix composites: Theoretical models and finite-element analysis. Composites Science and Technology, 61, 731-748.

Fornes, T. D., \& Paul, D. R. (2003). Modeling properties of nylon 6/clay nanocomposites using composite theories. Polymer, 44, 4993-5013.

Hbaieb, K., Wang, Q. X., Chia, Y. H. J., \& Cotterell, B. (2007). Modelling stiffness of polymer/clay nanocomposites. Polymer, 48, 901-909.

Hua, Y., \& Gu, L. (2012). Prediction of the thermomechanical behavior of particle-reinforced metal matrix composites. Composites Part B: Engineering, 45, 1464-1470.

Iisaka, K., \& Shibayama, K. (1978). Mechanical alpha-dispersion and interaction in filled polystyrene and polymethylmethacrylate. Journal of Applied Polymer Science, 22, 3135-3143.

Lassila, L. V. J., Nohrstrom, T., \& Vallittu, P. K. (2002). The influence of short-term water storage on the flexural properties of unidirectional glass fiber-reinforced composites. Biomaterials, 23, 2221-2229.

Lee, S. Y., Chiang, H. C., Lin, C. T., Huang, H. M., \& Dong, D. R. (2000). Finite element analysis of thermo-debonding mechanism in dental composites. Biomaterials, 21, 1315-1326.

Lee, S. H., Wang, S. Q., Pharr, G. M., \& Xu, H. T. (2007). Evaluation of interphase properties in a cellulose fiber-reinforced polypropylene composite by nanoindentation and finite element analysis. Composites Part A: Applied Science and Manufacturing, 38, 1517-1524.

Liu, H., \& Brinson, L. C. (2006). A hybrid numerical-analytical method for modeling the viscoelastic properties of polymer nanocomposites. Journal of Applied Mechanics: Transactions of the ASME, 73, 758-768.

Odegard, G. M., Clancy, T. C., \& Gates, T. S. (2005). Modeling of the mechanical properties of nanoparticle/polymer composites. Polymer, 46, 553-562.

Pierard, O., Friebel, C., \& Doghri, I. (2004). Mean-field homogenization of multi-phase thermo-elastic composites: A general framework and its validation. Composites Science and Technology, 64, 1587-1603.

Sevostianov, I., \& Kachanov, M. (2006). Homogenization of a nanoparticle with graded interface. International Journal of Fracture, 139, 121-127.

Sevostianov, I., \& Kachanov, M. (2007). Effect of interphase layers on the overall elastic and conductive properties of matrix composites. Applications to nanosize inclusion. International Journal of Solids and Structures, 44, $1304-1315$.

Sivasankaran, S., Sivaprasad, K., Narayanasamy, R., \& Iyer, V. K. (2010). Effect of strengthening mechanisms on cold workability and instantaneous strain hardening behavior during grain refinement of AA $6061-10 \mathrm{wt}$. $\% \mathrm{TiO}_{2} \mathrm{com}^{-}$ posite prepared by mechanical alloying. Journal of Alloys and Compounds, 507, 236-244.

Tian, M., Gao, Y., Liu, Y., Liao, Y. L., Hedin, N. E., \& Fong, H. (2008). Fabrication and evaluation of Bis-GMA/TEGDMA dental resins/composites containing nano fibrillar silicate. Dental Materials, 24, 235-243.

Tucker, C. L., \& Liang, E. (1999). Stiffness predictions for unidirectional short-fiber composites: Review and evaluation. Composites Science and Technology, 59, 655-671.

Turssi, C. P., Ferracane, J. L., \& Ferracane, L. L. (2006). Wear and fatigue behavior of nano-structured dental resin composites. Journal of Biomedical Materials Research Part B: Applied Biomaterials, 78B, 196-203.

Wei, C. Y., Srivastava, D., \& Cho, K. J. (2002). Thermal expansion and diffusion coefficients of carbon nanotube-polymer composites. Nano Letters, 2, 647-650.

Wetzel, B., Rosso, P., Haupert, F., \& Friedrich, K. (2006). Epoxy nanocomposites - Fracture and toughening mechanisms. Engineering Fracture Mechanics, 73, 2375-2398.

Widom, B. (1966). Random sequential addition of hard spheres to a volume. The Journal of Chemical Physics, 44 , 3888-3894.

Xia, Y., Zhang, F. M., Xie, H. F., \& Gu, N. (2008). Nanoparticle-reinforced resin-based dental composites. Journal of Dentistry, 36, 450-455.

$\mathrm{Yu}, \mathrm{B} ., \mathrm{Ahn}, \mathrm{J}$. S., Lim, J. I., \& Lee, Y. K. (2009). Influence of $\mathrm{TiO}_{2}$ nanoparticles on the optical properties of resin composites. Dental Materials, 25, 1142-1147.

Yu, S., Yang, S., \& Cho, M. (2009). Multi-scale modeling of cross-linked epoxy nanocomposites. Polymer, 50, 945-952.

Zhang, H., Zhang, Z., Friedrich, K., \& Eger, C. (2006). Property improvements of in situ epoxy nanocomposites with reduced interparticle distance at high nanosilica content. Acta Materialia, 54, 1833-1842. 